\title{
Degradable Cationic Polymer: A Class of Material to Address
}

\section{Bacteria Resistance}

\author{
Zhishuai Geng* \\ School of Chemistry \& Biochemistry, Georgia Institute of Technology, USA
}

*Corresponding author: Zhishuai Geng, School of Chemistry \& Biochemistry, Georgia Institute of Technology, 901 Atlantic Drive, Atlanta, Georgia, USA, 30332, Email: zhishuai@gatech.edu

Abbreviations: QACs: Quaternary Ammonium Compounds; AgNPs: Silver Nanoparticles; AMPs: Antimicrobial Peptides; MIC: Minimum Inhibition Concentration; MRSA: Methicillin Resistant Staphylococcus Aureus.

\section{Editorial}

Microbial infection is one of the major challenges to human health world-wide. Millions of deaths are caused by infectious diseases and malfunctioning medical device or equipment [1]. A variety of drugs have been developed to combat with all kinds of bacteria. However, due to the injudicious usage of these compounds, some bacteria have acquired resistance to most of them over years. Nowadays researchers are developing novel antimicrobial drugs to wipe out pathogenic bacteria before the emergence of resistance [2]. Thus, to address bacteria resistance towards antimicrobial is the essence of reducing infection.

There are several classes of most commonly used antimicrobial compounds: quaternary ammonium compounds (QACs), silver nanoparticles (AgNPs) and antimicrobial peptides (AMPs) in addition to traditionally used small-molecule antibiotics, such as penicillin. Traditional small-molecule antibiotics based on the inhibition of key enzymes are easily susceptible to the development and rapid dissemination of mutations in the target that confer resistance already in the last several decades and call for substitutions [3]. AgNPs is usually classified as more environmental unfriendly compounds due to their transformation product's long half-life, general accumulation and toxicity towards live organism [4]. QACs has also been used widely as broad spectrum antimicrobials since they target bacteria membrane which requires more genetic mutations [5].
Thus those small QACs have been touted as being difficult to generate resistance against, but this is proving to be incorrect by recent reports [6]. Commercial antimicrobial QACs have high stability and therefore inevitable accumulation in the environment. Long exposure of sublethal doses of QACs will drive the evolution and finally selected the strains immune to similar structures [7]. However, this mechanism has not gathered enough attention towards current development of new antimicrobials. AMPs, primarily also cationic in nature, are a class of larger molecules often used by microorganisms for defensive purposes. While it has been stated or supposed that resistance to these agents is harder to develop, their natural origin ensures that a variety of resistance mechanisms are known to exist or can be evolved [8].

Still, the ability of natural antimicrobial peptides to disrupt bacterial membrane integrity has inspired the development of many different types of charged synthetic oligomers or short polymers [9-11]. Because a great number of examples of these molecules successfully resisting natural defense mechanisms have been reported, they represent a promising approach towards antimicrobial applications.

The slow resistance development to charged oligomers/polymers may be attributed to its multivalent display of charged center. It has been reported that even for the small QACs, increasing charge center number from one to two will significantly reduce the probability of emergence of resistance. A variety of polycations have been tested as potential antimicrobials and the resistance development is also taken into consideration, including polyionenes [12,13] and polymers acquired from controlled polymerization with charged groups installed onto side chain [11,14-17]. 


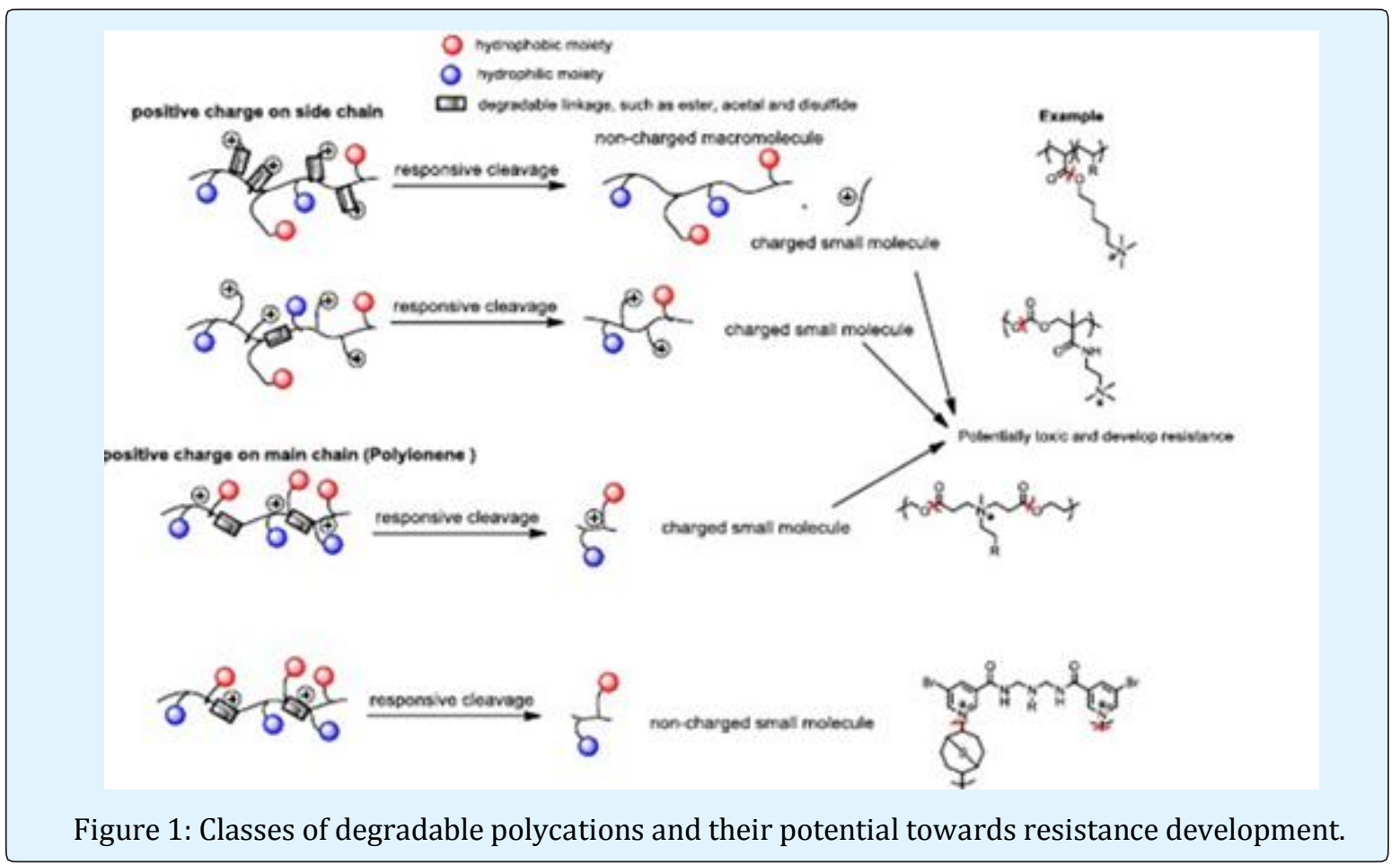

Currently the antimicrobial resistance has been characterized mainly through two dimensions. The most common way is to monitor MIC (minimum inhibition concentration) changes after repeated use of drugs at sub-lethal doses. This assay usually requires weeks of time for exposing certain concentration of healthy bacteria cells at logarithmic growth phase to a series of drug solution with varying concentration, thus not trivial to perform conventional antibiotics, such as ciprofloxacin, have become the most popular benchmark to be compared with novel cationic polymer in this method [12]. It has been clearly proved that cationic polymer has great potential in reducing the rate of resistance development, however, the comparison between different types of cationic polymers is still lacking. Recently a novel phenomenon has been reported in the investigation of resistance towards degradable Polycations [13]. Repeat exposure of E.coli cells towards degradable and nondegradable polycations led to two totally different results. Trying to mimic the reality of expansion of colonies in environment, cells have been exposed to polycations freshly made and kept in $37 \mathrm{oC}$ for 24 hours periodically. MIC of degradable polycations towards E.coli increased more slowly. And instead of cross-resistance, a crosssusceptibility has emerged, which is exposure of degradable polycation make E.coli make it more susceptible to other cationic molecules. For the first time, this phenomenon shed lights on a new direction to design of polymers for antimicrobial usage. According to similar findings in removal of $\mathrm{QAC}$ antimicrobial periodically, it seems the MIC increase indicating tolerance of certain antimicrobial is not permanent, called adaptation instead of resistance [18]. Thus, without more detailed mechanism study, it is difficult to conclude there are any genetic changes in the generations of cells treated with cationic compounds.

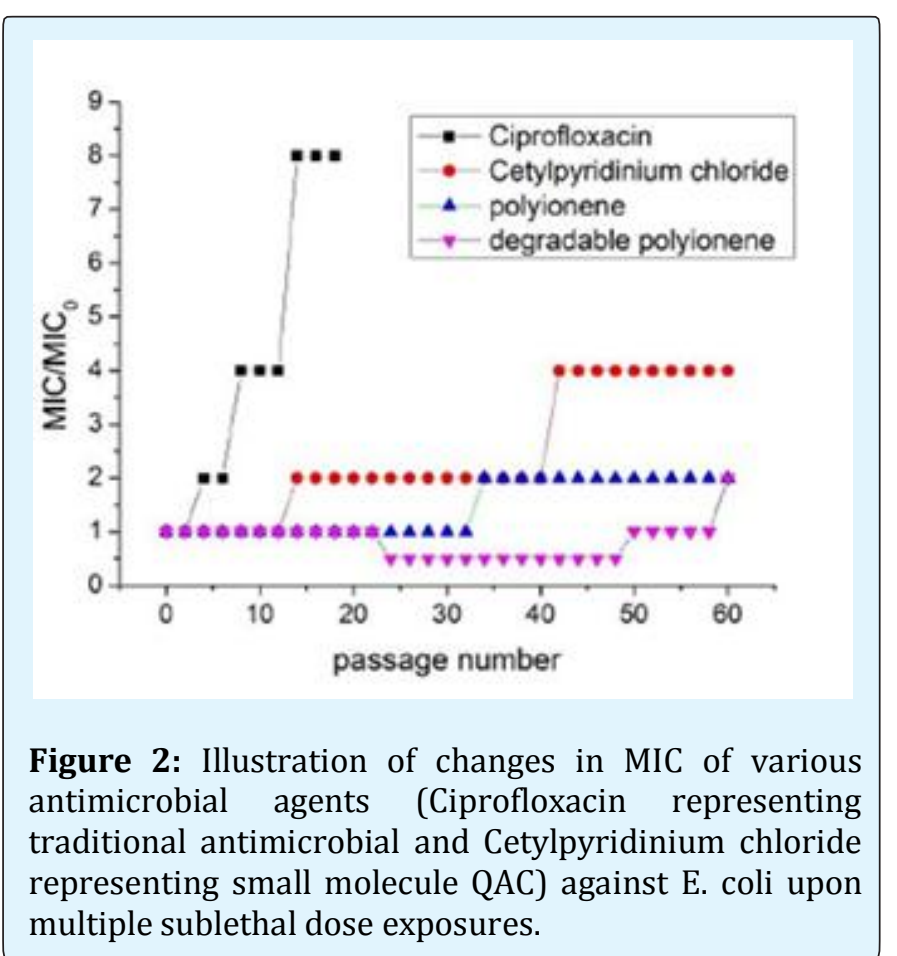

Another way to confirm the potential of antiresistance-development material/molecule is to examine its activity towards clinically isolated drugresistant bacterial strains, the most common example of which is MRSA (methicillin resistant Staphylococcus aureus). A variety of cationic compounds, both small molecules and macromolecules, have been confirmed to 


\section{Medicinal \& Analytical Chemistry International Journal}

be effective towards superbugs which can resist most common traditional antibiotics [19]. While the strains resisting QACs haven't been widely tested in new antimicrobial cationic materials yet. That provides a valuable dataset to control resistance development to this new class of antimicrobial polymer. Combining with the long term monitoring MIC, the test again strains with resistance already developed will become a standard way to approve and screen degradable cationic polymers towards the battle against bacteria.

Not only benefiting from slow resistancedevelopment, biodegradable cationic polymers are also facilely employed as building blocks for various biomaterials. Wound dressing materials usually were required to break down in a certain time window while maintaining antimicrobial function to prevent infections. Both natural and synthetic polymer based hydrogel could use the design of building in degradable cationic motif [20]. After the materials were broken down, the degraded product in low molecular weight was expected to be less toxic and easy to be metabolized. Another application is to coat degradable polycations on the surface of bulk material or device. Each layer of coating is decorated (covalently attached) or packed (via supramolecular interaction) with cationic antimicrobial components [13-21]. After one layer is exhausted or fouled, the environmental stimulated degradation will refresh the surface to be actively antimicrobial again without releasing majority of antimicrobials into outer environment to raise resistance concern.

Bacteria will inevitably find methods of resisting the antibiotics we develop, which have to be slowed down if not completely prevented to create a healthier and safer society. Among current strategies towards this tough problem, employment and development of next generation degradable cationic polymers had been paid little attention. However, it could lead us to a brighter future with the diversity in structure and high antimicrobial efficiency of polycations in addition to even lower resistance resulting from degradable linkage which chemists are able to contribute most.

\section{References}

1. Levy SB, Marshall B (2004) Antibacterial resistance worldwide: causes, challenges and responses. Nature Med 10(12): 122-129.

2. Taubes G (2008) The Bacteria Fight Back. Science 321(5887): 356-361.

3. Holmes AH, Moore LSP, Sundsfjord A, Steinbakk M, Regmi S, et al. (2016) Understanding the mechanisms and drivers of antimicrobial resistance. Lancet 387(10014): 176-187.

4. Choi O, Deng KK, Kim NJ, Ross L, Surampalli RY, et al. (2008) The inhibitory effects of silver nanoparticles, silver ions, and silver chloride colloids on microbial growth. Water Research 42(12): 3066-3074.

5. Denyer SP (1995) Mechanisms of action of antibacterial biocides. International Biodeterioration \& Biodegradation 36(3-4): 227245.

6. Jennings MC, Minbiole KPC, Wuest WM (2015) Quaternary Ammonium Compounds: An Antimicrobial Mainstay and Platform for Innovation to Address Bacterial Resistance. ACS Infect Dis 1(7): 288-303.

7. Tezel U, Pavlostathis SG (2011) Role of Quaternary Ammonium Compounds on Antimicrobial Resistance in the Environment. Antimicrobial Resistance in the Environment. John Wiley \& Sons, Inc, pp: 349-387.

8. Joo HS, Otto M (2015) Mechanisms of resistance to antimicrobial peptides in staphylococci. Biochim Biophys Acta 1848(11): 3055-3061.

9. Chakraborty S, Liu R, Hayouka Z, Chen X, Ehrhardt J, et al. (2014) Ternary Nylon-3 Copolymers as HostDefense Peptide Mimics: Beyond Hydrophobic and Cationic Subunits. J Am Chem Soc 136(41): 1453014535.

10. Lienkamp K, Madkour AE, Musante A, Nelson CF, Nüsslein K, et al. (2008) Antimicrobial Polymers Prepared by ROMP with Unprecedented Selectivity: A Molecular Construction Kit Approach. J Am Chem Soc 130(30): 9836-9843.

11. Kuroda K, DeGrado WF (2005) Amphiphilic Polymethacrylate Derivatives as Antimicrobial Agents. J Am Chem Soc 127(12): 4128-4129.

12. Liu S, Ono RJ, Wu H, Teo JY, Liang ZC, et al. (2017) Highly potent antimicrobial polyionenes with rapid killing kinetics, skin biocompatibility and in vivo bactericidal activity. Biomaterials 127: 36-48.

13. Geng Z, Finn MG (2017) Thiabicyclononane-Based Antimicrobial Polycations. Journal of the American Chemical Society 139(43): 15401-15406.

14. Lee SB, Koepsel RR, Morley SW, Matyjaszewski K, Sun Y, et al. (2004) Permanent, Nonleaching Antibacterial Surfaces. 1. Synthesis by Atom 


\section{Medicinal \& Analytical Chemistry International Journal}

Transfer

Radical

Biomacromolecules 5(3): 877-882.

15. Ilker MF, Nüsslein K, Tew GN, Coughlin EB (2004) Tuning the Hemolytic and Antibacterial Activities of Amphiphilic Polynorbornene Derivatives. J Am Chem Soc 126(48): 15870-15875.

16. Zhang J, Chen YP, Miller KP, Ganewatta MS, Bam M, et al. (2014) Antimicrobial Metallopolymers and Their Bioconjugates with Conventional Antibiotics against Multidrug-Resistant Bacteria. J Am Chem Soc 136(13): 4873-4876.

17. Xue Y, Xiao H, Zhang Y (2015) Antimicrobial Polymeric Materials with Quaternary Ammonium and Phosphonium Salts. Int J Mol Sci 16(2): 36263655.
18. Maxcy RB, Tiwari NP, Soprey PR (1971) Changes in Escherichia coli Associated with Acquired Tolerance for Quaternary Ammonium Compounds. Applied Microbiology 22(2): 229-232.

19. Nederberg F, Zhang Y, Tan JPK, Xu K, Wang H, et al. (2011) Biodegradable nanostructures with selective lysis of microbial membranes. Nature Chemistry 3(5): 409-414.

20. Pascual A, Tan JPK, Yuen A, Chan JMW, Coady DJ, et al. (2015) Broad-Spectrum Antimicrobial Polycarbonate Hydrogels with Fast Degradability. Biomacromolecules 16(4): 1169-1178.

21. Bieser AM, Thomann Y, Tiller JC (2011) ContactActive Antimicrobial and Potentially Self-Polishing Coatings Based on Cellulose. Macromolecular Bioscience 11(1): 111-121. 